HEFAT2014

$10^{\text {th }}$ International Conference on Heat Transfer, Fluid Mechanics and Thermodynamics

14 - 16 July 2014

Orlando, Florida

\title{
INVERSE VOF MESHLESS METHOD FOR EFFICIENT NON-DESTRUCTIVE THERMOGRAPHIC EVALUATION
}

\author{
Divo, E. ${ }^{1, *}$, Saad, H. ${ }^{1}$, Boetcher, S. ${ }^{1}$, Brown, J. ${ }^{2}$, and Kassab, A.J. ${ }^{3}$ \\ ${ }^{1}$ Department of Mechanical Engineering \\ ${ }^{2}$ Department of Civil Engineering \\ Embry-Riddle Aeronautical University \\ 600 South Clyde Morris Blvd, Daytona Beach, FL 32114 \\ *E-mail: divoe@erau.edu \\ ${ }^{3}$ Department of Mechanical and Aerospace Engineering \\ University of Central Florida \\ 4000 Central Florida Blvd, Orlando, FL 32816
}

\section{ABSTRACT}

A novel computational tool based on the Localized Radial-basis Function (RBF) Collocation (LRC) Meshless method coupled with a Volume-ofFluid (VoF) scheme capable of accurately and efficiently solving transient multi-dimensional heat conduction problems in composite and heterogeneous media is formulated and implemented. While the LRC Meshless method lends its inherent advantages of spectral convergence and ease of automation, the VoF scheme allows to effectively and efficiently simulate the location, size, and shape of cavities, voids, inclusions, defects, or de-attachments in the conducting media without the need to regenerate point distributions, boundaries, or interpolation matrices. To this end, the Inverse Geometric problem of Cavity Detection can be formulated as an optimization problem that minimizes an objective function that computes the deviation of measured temperatures at accessible locations to those generated by the LRC-VoF Meshless method. The LRC-VoF Meshless algorithms will be driven by an optimization code based on the Simplex Linear Programming algorithm which can efficiently search for the optimal set of design parameters (location, size, shape, etc.) within a predefined design space. Initial guesses to the search algorithm will be provided by the classical 1D semi-infinite composite analytical solution which can predict the approximate location of the cavity. The LRC-VoF formulation is tested and validated through a series of controlled numerical experiments. The proposed approach will allow solving the onerous computational inverse geometric problem in a very efficient and robust manner while affording its implementation in modest computational platforms, thereby realizing the disruptive potential of the proposed multidimensional high-fidelity non-destructive evaluation (NDE) method.

\begin{tabular}{lll}
\multicolumn{3}{l}{ NOMENCLATURE } \\
$T$ & {$[\mathrm{~K}]$} & Temperature \\
$c$ & {$[\mathrm{~J} / \mathrm{KgK}]$} & Specific heat capacity \\
$q$ & {$\left[\mathrm{~W} / \mathrm{m}^{2}\right]$} & Heat flux \\
$k$ & {$[\mathrm{~W} / \mathrm{mK}]$} & Thermal conductivity \\
$e$ & {$\left[\mathrm{~J} / \mathrm{m}^{2} \mathrm{Ks}{ }^{1 / 2}\right]$} & Thermal effusivity \\
$\rho$ & {$\left[\mathrm{kg} / \mathrm{m}^{3}\right]$} & Density \\
$x, y, z$ & {$[\mathrm{~m}]$} & Cartesian coordinates \\
$\hat{\beta}_{j}$ & {$[-]$} & Boundary condition coefficients \\
$\alpha_{j}$ & {$[-]$} & RBF expansion coefficients \\
$\chi_{j}(x)$ & {$[-]$} & Radial-basis functions (RBF) \\
$r_{j}(x)$ & {$[\mathrm{m}]$} & Euclidean distance from $x$ to $x_{j}$ \\
$d$ & {$[\mathrm{~m}]$} & RBF shape parameter \\
$x_{c}$ & {$[\mathrm{~m}]$} & Topology data center \\
$\{L\}$ & {$[-]$} & Derivative interpolation vector \\
$s$ & {$[-]$} & Volume-of-Fluid (VoF) parameter \\
$S(z)$ & {$[-]$} & Objective function \\
$N_{m}$ & {$[-]$} & Number of measurements \\
$N_{r}$ & {$[-]$} & Number of cluster rays
\end{tabular}




\section{INTRODUCTION}

In 2010, the Federal Highway Administration estimated that $89.5 \%$ ( $\$ 12.8$ billion) [1] of the total capital outlay for bridges in the U.S. was applied to replacement or rehabilitation. As the large proportion of the nation's bridge inventory constructed between 1950 and 1970 continues to age, with one-half of all bridges in the U.S. constructed before 1964 [2], the total spending required to maintain the functionality of our nation's bridge infrastructure has increased an average of $7.3 \%$ per year between 2000 and 2008 [1]. For over three decades, considerable research has been conducted in the field of strengthening existing reinforced concrete (RC) structures with fiberreinforced polymer (FRP) composite materials [3]. This research has resulted in design standards and specifications that engineers can apply to achieve a wide variety of strengthening objectives [4]-[6], such as increasing the shear and flexural capacity of reinforced concrete (RC) members and providing additional confinement for RC columns. A primary advantage of external strengthening with FRP composites is the on-site flexibility that is afforded by these materials (Figure 1). Reinforced concrete strengthening applications often utilize the wet lay-up method, which involves saturating dry fibers on-site with a polymer matrix material (typically epoxy) and applying the wetted composite directly to the concrete surface. Mechanical and chemical bond is established between the concrete and the composite during curing and stresses are transferred from the concrete to the composite via shear through the bondline as the structure is loaded.

The flexibility afforded by the wet lay-up method also makes these systems vulnerable to installation defects. The extent to which installation defects manifest as long-term durability concerns is not well understood, and there is no universally accepted nondestructive evaluation method for monitoring the long-term health and durability of these systems in the field. Thermal imaging techniques have shown great promise for identifying the presence/absence of defects in a qualitative sense [7]-[9]. Quantitative methods have also been employed in laboratory settings to demonstrate the potential for defect characterization [10]-[11]. There is a great need, however, for a rapid, robust method for fully characterizing the location, size, depth, and material composition of any anomalies that are encountered during an infrared thermography inspection.

Figure 1 represents a typical FRP strengthening application for an interstate overpass that was damaged in a collision with an over-height vehicle outside of Jacksonville, Florida. The qualitative thermal images indicated numerous installation defects, but, without employing more rigorous quantitative methods to characterize the nature of the anomalies, it is impossible to determine the underlying cause of the defects or their potential impact on the long-term efficacy of the repair. The proposed research will lay the foundation for new research thrusts in the area of FRP durability in civil infrastructure applications. A rapidly deployable method that provides a comprehensive description of the nature of encountered defects is the first step towards advancing materials processing techniques to minimize the occurrence of defects in the first place. For systems that are currently in-service, the proposed research will lead to more effective means for structural health monitoring and ensuring that installed systems perform as expected.

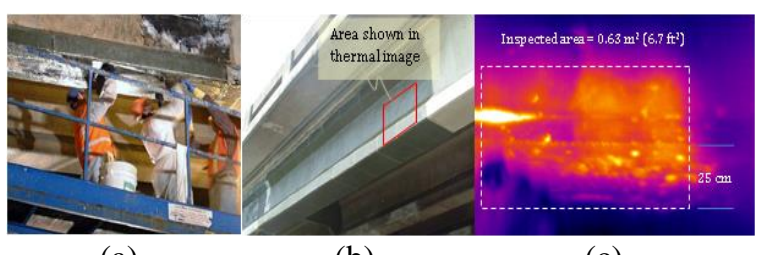

(a)

(b)

(c)

Figure 1 Application of FRP composite to strengthen existing interstate overpass. a) Workers applying carbon-fiber composite. b) Completed project. c)

Qualitative thermal imaging results obtained during non-destructive evaluation.

Within the family of inverse heat transfer problems [12], the inverse geometric problem finds its application in the nondestructive evaluation of subsurface flaws and cavities. Here, the governing equation, the thermophysical properties, the initial condition, the boundary conditions, and the portion of the geometry which is exposed, are all known. However, the portion of the problem geometry that is hidden from view is unknown and to be determined with the help of an overspecified (Cauchy) condition at the exposed surface; see Figure 2. Specifically, the surface temperature and heat flux are given at the exposed surface and the geometry of the cavity(ies) that generated the measured temperature footprint is to be determined. The boundary condition at the cavity side is specified as either homogeneous or nonhomogeneous first, second, or third kind of boundary condition. Solution of the inverse geometric problem can be undertaken by considering either the transient or steady-state thermal response of the system subjected to a thermal load. Consequently, there are two general categories of techniques for the solution of the inverse geometric problem: transient based (also known as thermal wave imaging methods) [13]-[14] and steady-state based (also known as infrared computerized axial tomography, IR CAT) [15]. In the case of the steady- 
state inverse geometric problem Ramm [16] demonstrates mathematically that the solution is unique for media with constant thermal conductivity.

The inverse geometric problem, which has been solved by a variety of numerical methods [17], and its closely related shape optimization problem [18], are arguably the most computationally intensive of all inverse heat transfer problems. This is due to their inherent nature, regardless of whether a numerical or analytical approach is taken to solve the associated direct problem, which requires a complete regeneration of the mesh as the geometry evolves. Moreover, the continuous evolution of the geometry itself poses certain difficulties in arriving at analytical or numerical sensitivity coefficients [19]-[20] for gradient-based optimization approaches and in the updates of the subsurface geometry(ies) and associated mesh(es), particularly in three dimensions, whether using domain-meshing methods such as finite-element or finite-volume methods, or boundary-meshing methods such as boundary elements [21]-[22], which have been developed extensively by the co-authors Divo and Kassab along with their research team [23].

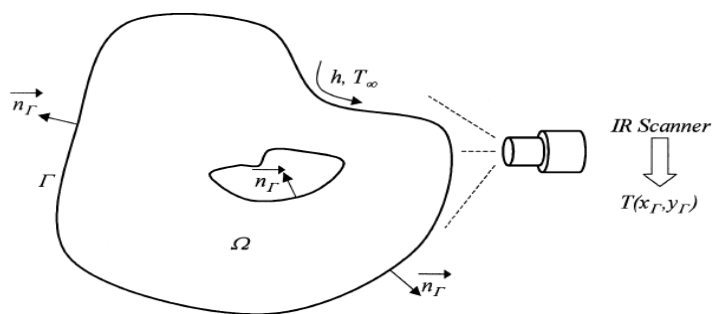

Figure 2 Problem setup using IR scanner to measure thermal footprint at the exposed boundary.

An efficient approach was introduced by Divo and Kassab [24] where singularity clusters were employed in a boundary element method (BEM) heat conduction formulation to simulate the presence of subsurface cavities in 2D and 3D geometries. The efficiency of this approach comes from the fact that the problem geometry does not need to be regenerated during the search process. Instead, the search is performed for the location, distribution, and strength of singularity clusters that act as voids within the medium. This allowed for accurate and efficient identification of subsurface cavities without the need of regenerating geometries or BEM interpolation matrices. This technique was later extended by Ojeda, Divo, and Kassab [25] for biomechanical applications of cavity detection in cortical bones. In this case, the deformation field difference with respect to a measured field at the exposed boundaries was minimized by using an elastostatics BEM code and employing a variety of differently shaped anchored grid patterns (AGP) that adapt to the shape of the internal cavity using the efficient singularity superposition idea.

While finite-element methods (FEM), finitevolume methods (FVM), and boundary-element methods (BEM) have been developed to a mature stage such that they are now utilized routinely to model complex multi-physics problems, they require significant effort in mesh generation and problem setup. Meshless methods are a relative newcomer to the field of computational methods, and the term "Meshless Methods" refers to the class of numerical techniques that rely on either global or localized interpolation on non-ordered spatial point distributions. As such, there has been much interest in the development of these techniques as they have the hope of reducing the effort devoted to model preparation [26]-[28]. The approach finds its origin in classical spectral or pseudo-spectral methods [29][30] that are based on global orthogonal functions such as Legendre or Chebyshev polynomials requiring a regular nodal point distribution. In contrast, Meshless methods use a nodal or point distribution that is not required to be uniform or regular due to the fact that most such techniques rely on global radial-basis functions (RBF) [31]-[34]. RBF have proved quite successful in their application to an earlier mesh-reduction method, namely the dual reciprocity boundary element method (DRBEM). However, global RBF-based Meshless methods have some drawbacks, including poor conditioning of the ensuing algebraic set of equations, which can be addressed to some extent by domain decomposition and appropriate pre-conditioning [35]-[37]. Moreover, care must be taken in the evaluation of derivatives in global RBF-based Meshless methods. Although very promising, these techniques can also be computationally intensive. Recently, localized collocation Meshless methods [38] have been proposed to address many of the issues posed by global RBF Meshless methods.

In a series of recent publications [39]-[43], Divo, Kassab, and their group have developed a Localized Collocation Meshless Method (LCMM) based on Radial-Basis Function (RBF) interpolation for modeling of coupled viscous fluid flow, heat transfer problems, and fluid-structure interaction problems. The LCMM features Hardy Multiquadrics RBF augmented by polynomial expansions over a local topology of points for the sought-after unknowns with an efficient formulation for computing the interpolations in terms of vector products. This approach is applicable to explicit or implicit time marching schemes as well as steady-state iterative methods. The LCMM technique lends itself very well to parallel computations and has been shown to be 
computationally more efficient than a comparative finite volume method (FVM) code whilst affording the distinct advantage of solving the partial differential conservation field equations of fluid flow and heat transfer on a non-ordered set of points. The method has been extensively verified against benchmarks and validated finite volume codes for several cases. This technique has been implemented in the solution of inverse heat transfer problems [44] as well as shape optimization problems [45]. An alternative approach to the cavity detection problem using Meshless methods was proposed by Karageorghis et al [46] by formulating a moving pseudo-boundary method of fundamental solutions to detect voids and boundary locations.

The need arises for an efficient technique that avoids the implicit requirement of performing completely new solutions as the geometry is sought while offering the possibility for automation and the robustness of predicting the location, size, and shape of cavities, voids, inclusions, defects, or deattachments within the conducting media.

\section{THE LOCALIZED RBF COLLOCATION (LRC) MESHLESS METHOD}

The Meshless formulation begins by defining a set of data centers, $N C$, comprised of points on the boundary, $N B$, and points on the interior, $N I$. These data centers will serve as collocation points for the localized expansion of the different field variables in the domain, $\Omega$, and on the boundary, $\Gamma$, see Figure 3 . The essential difference between boundary points and internal points is simply that boundary conditions will be applied at the first while governing equations will be applied at the last.

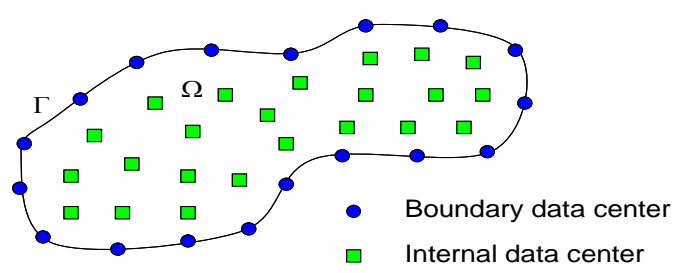

Figure 3 Scattered point distribution in a generalized domain

To illustrate the Meshless formulation the diffusion equation for the temperature, $T$, in a generalized coordinate system, $x$, and time, $t$, will be taken into consideration as the governing equation valid in the domain, $\Omega$, with constant conductivity, $k$, density, $\rho$, and specific heat capacity, $c$, as:

$$
\frac{\partial T}{\partial t}(x, t)=\frac{k}{\rho c} \nabla^{2} T(x, t)
$$

In addition, a set of generalized boundary conditions on the boundary, $\Gamma$, are given by:

$$
\hat{\beta}_{1} \frac{\partial T}{\partial n}+\hat{\beta}_{2} T=\hat{\beta}_{3}
$$

Where $\hat{\beta}_{1}, \hat{\beta}_{2}$, and $\hat{\beta}_{3}$ are imposed coefficients of $(x, t)$ that dictate the boundary condition type and constraint values. A linear localized expansion over a group or topology of influence points, $N F$, around each data center is sought such that:

$$
T(x)=\sum_{j=1}^{N F} \alpha_{j} \chi_{j}(x)+\sum_{j=1}^{N P} \alpha_{j+N F} P_{j}(x)
$$

The terms $\alpha_{j}$ represent the unknown expansion coefficients while the terms $\chi_{j}(x)$ are expansion functions defined a-priori. While $N P$ is a number of additional polynomial functions, $P_{j}(x)$, added to the expansion to guarantee that constant and linear fields can be retrieved by the expansion exactly. Notice that the time dependency has been dropped as a different expansion will be performed for each time level and, therefore, the expansion coefficients, $\alpha_{j}$, will vary as time progresses. The time dependency of the expansion coefficients, $\alpha_{j}$, is implicit as these are not explicitly computed as time progresses but instead algebraic manipulations of the expansion allow to express the derivative fields directly in terms of the field variable values within the influence topology, as it will be shown later. The expansion functions $\chi_{j}(x)$ are selected as the Inverse Hardy Multiquadrics Radial-basis functions (RBF), defined as:

$$
\chi_{j}(x)=\left[\left(\frac{r_{j}(x)}{d}\right)^{2}+1\right]^{-\frac{1}{2}}
$$

Here, the term $r_{j}(x)$ is the Euclidean distance from any point $x$ to an expansion point $x_{j}$, while the term $d$ is a shape parameter. The larger this shape parameter $d$ the flatter the expansion function becomes and therefore the derivative field becomes smoother. However, the value of the shape parameter $d$ cannot be increased indefinitely as the resulting coefficient matrix from the collocation process becomes ill-conditioned. A simple search process is performed to determine the optimal value of this shape parameter $d$ for each localized expansion. The behavior of this RBF expansion function has been widely studied in the literature [32]-[37]. 
The selection of an influence region or localized topology of expansion around each data center is easily accomplished by a circular (spherical in 3D) search around each data center. The search is automated to guarantee that a minimum number of points is included and additional criteria, such as including all directions around internal data centers, are met. In addition, this search must guarantee that topologies around boundary data centers do not include opposing boundaries or points around a reentry corner. Figure 4 shows a typical collocation topology for a non-uniform point distribution.

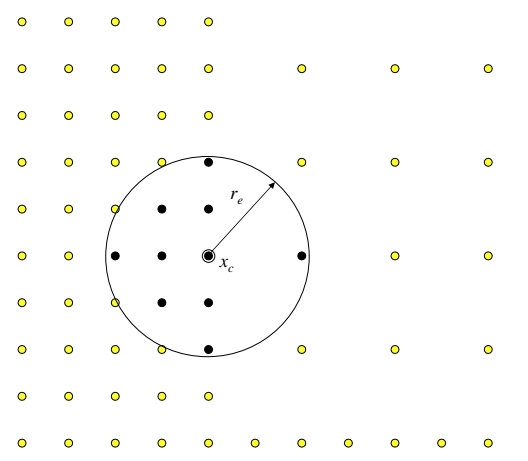

Figure 4 Collocation topology selection on a nonuniform point distribution.

The collocation of the known temperature field (from previous time level or iteration step) at the points within the localized topology, can be expressed in matrix-vector form as: $\{T\}=[C]\{\alpha\}$, and, therefore, the expansion coefficients can be determined as: $\{\alpha\}=[C]^{-1}\{T\}$. Where the resulting collocation matrix is given by:

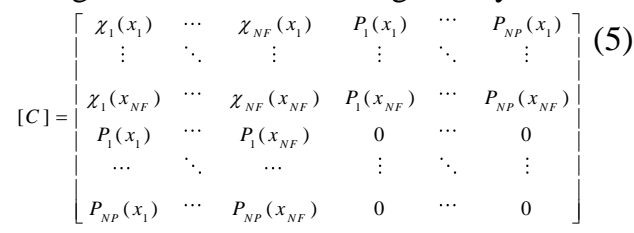

And the right-hand side known vector is augmented as:

$$
\{T\}=\left\{T\left(x_{1}\right) \ldots T\left(x_{N F}\right) 0 \ldots 0\right\}_{1, N F+N P}^{T}
$$

Note that the polynomial-augmented matrix in Eqn. (5) guarantees constant and linear (or as high as the polynomial order employed) temperature fields to be expanded exactly. The augmentation of the temperature vector in Eqn. (6) with values of zero does not indicate zero temperatures but rather the dimensional consistency with the expansion matrix. The real advantage of the localized collocation approach is capitalized in the way the derivatives of the field variable are calculated at the data center, $x_{c}$ of each topology. For instance, any linear differential operator, $L$, can be applied over the localized expansion equation as:

$$
L T\left(x_{c}\right)=\sum_{j=1}^{N F} \alpha_{j} L \chi_{j}\left(x_{c}\right)+\sum_{j=1}^{N P} \alpha_{j+N F} L P_{j}\left(x_{c}\right)
$$

Or, in matrix-vector form: $L T_{c}=\left\{L_{c}\right\}^{T}\{\alpha\}$, where the derivative expansion vector $\left\{L_{c}\right\}$ is given as:

$$
\left\{L_{c}\right\}=\left\{\begin{array}{l}
L \chi_{1}\left(x_{c}\right) \ldots \\
L \chi_{N F}\left(x_{c}\right) L P_{1}\left(x_{c}\right) \ldots L P_{N P}\left(x_{c}\right)
\end{array}\right\}^{T}
$$

Substitution of the expansion coefficients, $\{\alpha\}$, leads to: $L T_{c}=\left\{L_{c}\right\}^{T}[C]^{-1}\{T\}$, and defining the derivative interpolation vector $\{L\}$ as:

$$
\{L\}^{T}=\left\{L_{c}\right\}^{T}[C]^{-1}
$$

Leads to the final expression:

$$
L T_{c}=\{L\}^{T}\{T\}
$$

The coefficients of the derivative interpolation vector $\{L\}$ of size $(N F, 1)$ directly retrieve the value of the derivative of the temperature field $\{T\}$ at the data center of the topology $x_{c}$. Therefore, evaluation of the temperature derivatives at each of the data centers $x_{c}$ is provided by a simple inner product of two small vectors: $\{L\}$ which can be prebuilt and stored at a setup stage of the problem as it is only dependent on geometry and point distribution, and $\{T\}$, which is the updated temperature field in the topology of the data center.

Furthermore, imposition of the generalized boundary conditions in Eqn. (2), at the boundary data centers, $x_{c}$, can be accomplished in a similar fashion. To aid the boundary interpolation an additional set of internal points that "shadow" each boundary point in the direction of the normal vector into the domain, as seen in Figure 5, are included in the point distribution and used to directly approximate the normal derivatives at each boundary data center.

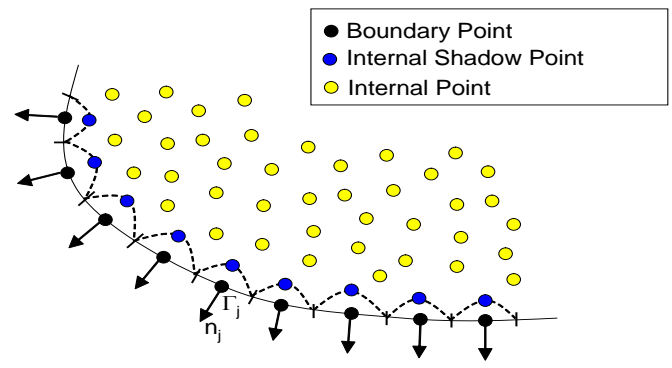

Figure 5 Distribution of internal shadow points to compute normal derivatives. 
This localized expansion approach reduces the burden of the more common global interpolation methods [35] by expanding the field variable locally around each data center to obtain its derivatives that are then used in time-marching or iterative schemes. This approach yields the generation of multiple but small derivative interpolation vectors that can be prebuilt and stored at a setup stage of the problem as they are only dependent on geometry and point distribution. Additional interpolation vectors for Moving Least-Square (MLS) smoothing and Upwinding schemes can be pre-computed and stored in an analogous fashion, see [40]-[43].

\section{THE VOLUME-OF-FLUID (VOF) METHOD}

The Volume-of-Fluid (VoF) method was introduced by Hirt and Nichols [47] to approximate the behavior of two-phase non-mixing fluid flow problems by implicitly tracing the interface between the two dissimilar fluids through the transport of a continuous variable $s$ that quantifies the content of one of the fluids ( $s=1$ ) (and absence of the other one) or the absence of this fluid $(s=0)$ (and content of the other one) as:

$$
\frac{\partial s}{\partial t}+(\vec{V} \cdot \nabla) s=0
$$

Therefore, the VoF parameter $s$ is used to postdetermine the location of the interface between the two phases, $(s=0.5)$. This approach offers the great advantage that a two-phase flow problem can be modeled in a single domain through a single set of governing equations while the $\mathrm{VoF}$ parameter $s$ is used as a weighting factor for the thermo-physical properties of the two fluids as, for instance, in the case of the thermal conductivities $k^{1}$ and $k^{2}$ :

$$
k=(1-s) k^{1}+s k^{2}
$$

One of the premises of this research is that the presence of a cavity within a thermally conducting medium can be simulated and approximated using a static version of the VoF method, where the parameter $s$ is not transported through the static field $\vec{V}=0$ but simply fixed at a value $s=0$ at the hypothetical location of the cavity and $s=1$ elsewhere. And, therefore, there is no need to model the actual geometry of the cavity.

\section{NUMERICAL IMPLEMENTATION}

To illustrate this approach, the LRC Meshless method coupled with the VoF method is tested in a composite domain made of a $(1 \mathrm{~m} \times 1 \mathrm{~m})$ Concrete block $\left(\rho=2300 \mathrm{~kg} / \mathrm{m}^{3}, \quad c=880 \mathrm{~J} / \mathrm{kgK} \quad\right.$ and $k=1.4 \mathrm{~W} / \mathrm{mK})$ with an attached $(0.1 \mathrm{~m} \times 1 \mathrm{~m})$ epoxy layer $\left(\rho=1000 \mathrm{~kg} / \mathrm{m}^{3}, c=1000 \mathrm{~J} / \mathrm{kgK}\right.$, and $k=14 W / m K$ ) with perfect thermal contact. The composite domain is at an initial temperature $T_{i}=20^{\circ} \mathrm{C}$ and heated with a constant heat flux $q_{0}=1000 \mathrm{~W} / \mathrm{m}^{2}$ through the exposed epoxy wall while insulated elsewhere. A uniform LRC Meshless point distribution with $\Delta x=\Delta y=0.01 \mathrm{~m}$ was employed to model this problem as shown in Figure 6 along with the resulting temperature field after $1000 \mathrm{~s}$ of heating.
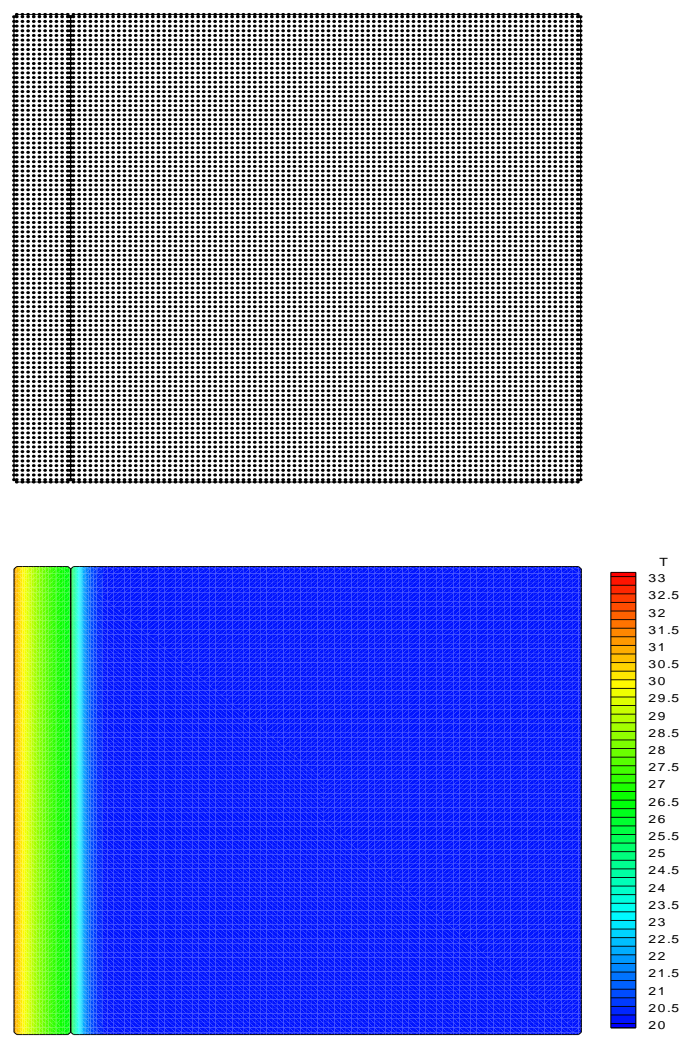

Figure 6 Meshless point distribution and resulting temperature field after 1000 s of heating

Furthermore, a cavity is modeled by a $(0.01 \mathrm{~m} \times 0.2 \mathrm{~m})$ sliver centered at the contact midpoint of the composite. In order to simulate this cavity using the VoF approach, the LRC Meshless points at the location of the expected cavity were imposed with a VoF parameter $s=0$, while a value of $s=1$ was imposed elsewhere. The VoF parameter $s$ essentially weights the thermo-physical properties of the epoxy with those of Air $\left(\rho=1.2 \mathrm{~kg} / \mathrm{m}^{3}, \quad c=1000 \mathrm{~J} / \mathrm{kgK}, \quad\right.$ and $k=0.05 \mathrm{~W} / \mathrm{mK}$ ) which acts as an almost perfect 
insulator $(k \sim 0)$ but capable of diffusing energy $(\alpha=k / \rho c)$ better than the epoxy. The resulting LRC Meshless temperature field after 1000 s of heating is shown in Figure 7 for the case of (a) the actual cavity and (b) the VoF-simulated cavity. Notice that the temperature footprint provided by the VoFsimulated cavity is qualitatively very similar to that provided by the actual cavity. This feature is revealed in more detail in Figure 8 where the temperature profiles at the exposed wall are compared after 1000s of heating. Notice that the profiles are in very close agreement, demonstrating the capacity of the $\mathrm{VoF}$ method to simulate not only the presence of a cavity but also its location, size, and shape, as these features are captured by the cluster of LRC Meshless points that were imposed with the $\mathrm{VoF}$ parameter $s=0$. Figure 8 also shows the constant temperature profile produced by the attached domain (or composite in perfect thermal contact), revealing that there is sufficient sensitivity $\left(\sim 2^{\circ} \mathrm{C}\right)$ to detect the thermal footprint produced by a de-attachment or cavity by standard measurement techniques such as infrared (IR) thermography.
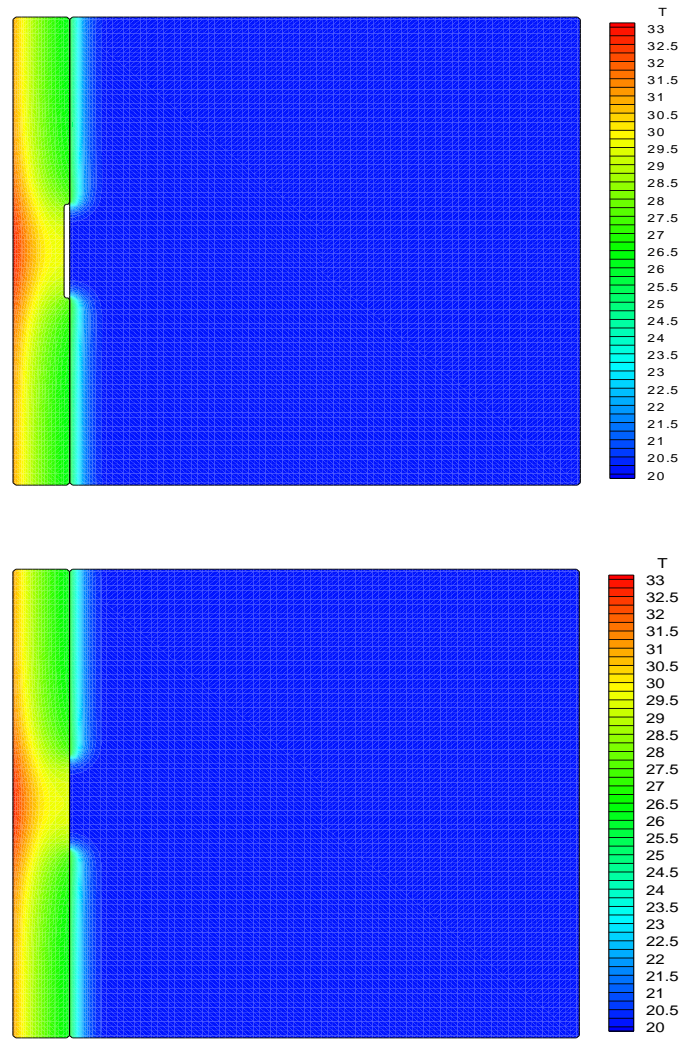

Figure 7 LRC Meshless temperature field after 1000s of heating. (a) Actual cavity and (b) VoFsimulated cavity.

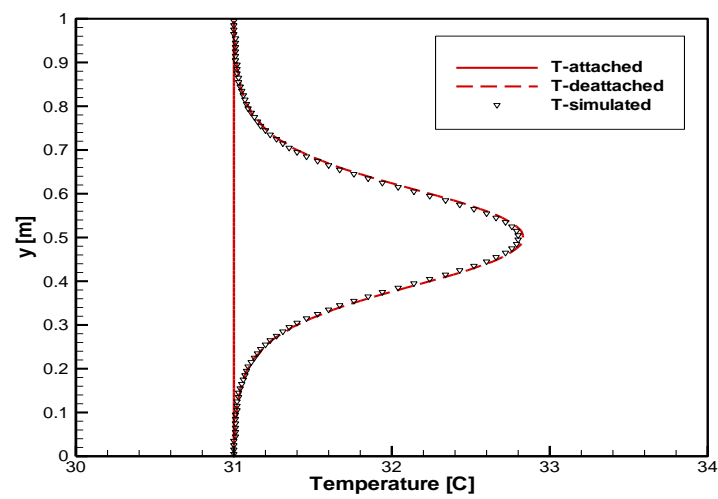

Figure 8 Temperature distribution on left-hand side wall after $\mathrm{t}=1000 \mathrm{~s}$ of heating.

\section{THE INVERSE PROBLEM FORMULATION}

The inverse problem of determining the location, size, and shape of the cavity may be formulated as an optimization problem whose objective is to minimize a function that computes the standard deviation between the LRC-VoF-computed temperatures $T_{i}$ at the exposed boundaries and the temperatures acquired through IR measurements $\hat{T}_{i}$. This can be expressed as a least-squares function over a finite number of measurement locations $N_{m}$, or:

$$
S(z)=\sqrt{\frac{1}{N_{m}} \sum_{i=1}^{N_{m}}\left[T_{i}(z)-\hat{T}_{i}\right]^{2}}
$$

The measurement locations may be fixed or movable throughout the solution process. The solution may also be expected to be sensitive to the location and number $N_{m}$ of measurements. A full sensitivity analysis will be performed in a follow-up work to optimize the number and location of such measurements. The objective function $S$ depends on a number of geometric parameters $z$ that define the location, size, and shape of the cluster of LRC Meshless points that are imposed with a VoF value $s=0$, i.e. a simulated cavity. For instance, in $2 \mathrm{D}$, the cluster may be generally defined by an anchored Cubic spline set centered at $\left(x_{o}, y_{o}\right)$ with a number of rays $N_{r}$ each extending a distance $r_{i}$ from the center, expressed in polar coordinates as:

$$
\begin{aligned}
& r(\theta)=M_{i-1} \frac{\left(\theta_{i}-\theta\right)^{3}}{6 \Delta \theta_{i}}+M_{i} \frac{\left(\theta-\theta_{i-1}\right)^{3}}{6 \Delta \theta_{i}} \\
& +\left(r_{i-1}-\frac{M_{i-1} \Delta \theta_{i}^{2}}{6}\right) \frac{\theta_{i}-\theta}{\Delta \theta_{i}} \\
& +\left(r_{i}-\frac{M_{i} \Delta \theta_{i}^{2}}{6}\right) \frac{\theta-\theta_{i-1}}{\Delta \theta_{i}}
\end{aligned}
$$


Where $M_{i}$ is the value of the second derivative of the spline at the node $i$, and $\Delta \theta_{i}$ is the angular spread of each spline, i.e. $\Delta \theta_{i}=\pi / 4$ for $N_{r}=8$. Requiring that the first and second derivatives are continuous at all nodes of the spline set results in a simple 8-dof tri-diagonal system for the values of $M_{i}$

A sample anchored Cubic spline set is shown in Figure 9 for $N_{r}=8$. All the LRC Meshless points that lie within the resulting anchored Cubic spline set will then be imposed with a VoF parameter value of $s=0$.

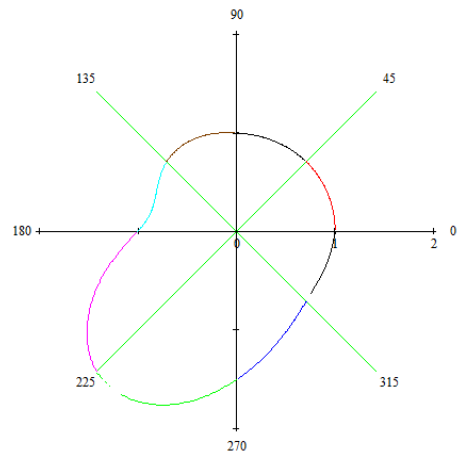

Figure 9 Anchored Cubic spline set for $N_{r}=8$

Therefore, the parameters $z$ in the objective function are the coordinates of the center and the size $r_{i}$ of the $N$ rays of the cubic (in 2D) or bi-Cubic (in 3D) spline set. The minimization of the objective function $S(z)$ may be accomplished by a nongradient based method such as Nelder and Mead's linear programming Simplex search method [48]. The simplex method is defined by the number of unknown parameters that are of interest. The "Simplex" is a geometrical figure with $N+1$ points and $N$ dimensions, where $N$ is the number of parameters. For example, in a 2-dof problem the Simplex is a triangle, and in a 3-dof problem the simplex is a tetrahedron. As for the solution marching logic, the Simplex can be manipulated by expansion, contraction, and reflection. These three tools are utilized to move the solution with the highest error around the solutions of low error to find a new minimum. In essence, the Simplex is moved in a downhill manner until a tolerance between solutions is reached. The current work attempts to establish the feasibility of the Simplex search process aided by the LRC-VoF scheme. To this end, the computational framework was established however, its implementation is currently under way. A follow-up work will include full detail about the numerical implementation of the Simplex search method including the convergence or stopping criteria.

The Simplex search process may be aided by a good initial guess provided by the classical 1D temperature distribution solution. For instance, for the case of the composite domain studied in the previous section, the $1 \mathrm{D}$ temperature evolution at the heated wall of a finite layer of length $L$ and properties $k_{0}, \rho_{0}$, and $c_{0}$, attached to a semiinfinite substrate with properties $k_{1}, \rho_{1}$, and $c_{1}$ is given by (recall that the thermal diffusivity $\alpha=k / \rho c$ and the thermal effusivity $e=\sqrt{k \rho c}$ ):

$$
\begin{aligned}
& \Delta T(t)=\frac{2 q_{0}}{k_{0}} \sqrt{\frac{\alpha_{0} t}{\pi}} \\
& \left\{\begin{array}{l}
1_{n=1}^{\infty}\left(\frac{e_{1}-e_{0}}{e_{1}+e_{0}}\right)^{n} \\
\left.\left\{\exp \left(-\frac{n^{2} L^{2}}{\alpha_{0} t}\right)-\frac{n L \sqrt{\pi}}{\sqrt{\alpha_{0} t}} \operatorname{erfc}\left(\frac{n L}{\sqrt{\alpha_{0} t}}\right)\right]\right\}
\end{array}\right.
\end{aligned}
$$

To predict the temperature response produced by a de-attachment, the properties of the substrate material index $l$ can be substituted for those of air (for example). The temperature responses for the problem described in the previous section with $q_{0}=1000 \mathrm{~W} / \mathrm{m}^{2}$ are shown in Figure 10 . Here, the transient response at the center point of the heated wall $(y=0.5 m)$ provided by the LRC Meshless solution with the actual cavity and the VoF-simulated cavity are shown in comparison with the temperature response provided by the 1D solution in Eqn. (15). Notice that the 1D solution tends to under-predict the actual temperature response due to its inability to factor in the actual size of the de-attachment.

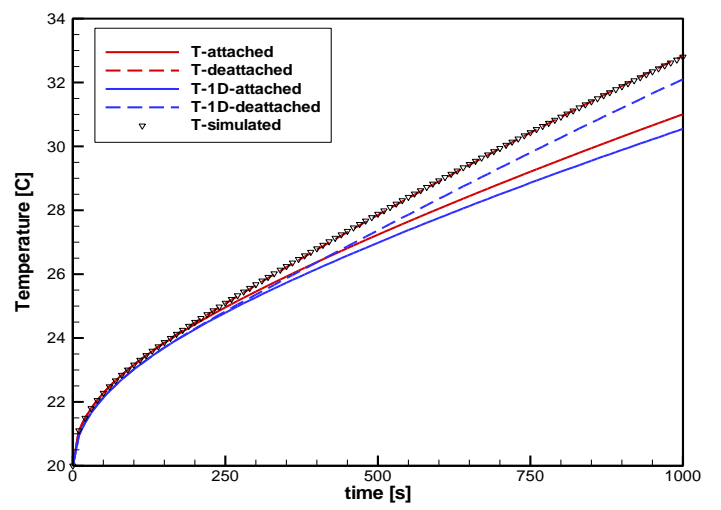

Figure 10 Temperature response at the mid-point of the heated wall provided by the LRC-VoF Meshless and the $1 \mathrm{D}$ solutions. 


\section{CONCLUSIONS}

A novel computational tool based on the Localized Radial-basis Function (RBF) Collocation (LRC) Meshless method coupled with a Volume-ofFluid (VoF) scheme capable of accurately and efficiently solving transient multi-dimensional heat conduction problems in composite and heterogeneous media is formulated and implemented. While the LRC Meshless method lends its inherent advantages of spectral convergence and ease of automation, the VoF scheme allows to effectively and efficiently simulate the location, size, and shape of cavities, voids, inclusions, defects, or de-attachments in the conducting media without the need to regenerate point distributions, boundaries, or interpolation matrices. To this end, the Inverse Geometric problem of Cavity Detection is formulated as an optimization problem that minimizes an objective function that computes the deviation of measured temperatures at accessible locations to those generated by the LRCVoF Meshless method. The LRC-VoF Meshless algorithms is driven by an optimization code based on the Simplex Linear Programming algorithm which can efficiently search for the optimal set of design parameters (location, size, shape, etc.) within a predefined design space. Initial guesses to the search algorithm are provided by the classical 1D semiinfinite composite analytical solution which can predict the approximate location of the cavity. The LRC-VoF formulation is tested using numerical experiments that reveal a high degree of accuracy and serve to validate the approach.

\section{REFERENCES}

[1] U.S. House of Representatives Committee on Transportation and Infrastructure, Memo to House members of Subcommittee on Highways and Transit, June 4, 2008.

[2] Federal Highway Administration, "2010 Status of the Nation's Highways, Bridges, and Transit: Conditions and Performance," Report to the United States Congress, 2010.

[3] L. C. Bank, Composites for Construction. Hoboken, New Jersey: John Wliey and Sons, Inc., 2006.

[4] American Concrete Institute (ACI) Committee 440, "Guide for the design and construction of externally bonded FRP systems for strengthening concrete structures," American Concrete Institute, ACI 440.2R08, Farmington Hills, Michigan, 2008.

[5] International Federation for Structural Concrete (FIB), "Retrofitting of concrete structures by externally bonded FRPs, with emphasis on seismic applications," International Federation for Structural Concrete, FIB Bulletin 35, Lausanne, Switzerland, 2006.

[6] Darby, A., Ibell, T. and Clarke, J., TR55 "Design guidance for strengthening concrete structures using fibre composite materials." London: The Concrete Society, 2004.
[7] Brown, J. and Hamilton III, H.R.. "Investigation of Heating Methods and Detection Limits for Infrared Thermography Inspection of FRP Composites Used to Strengthen Reinforced Concrete", ACI Materials Journal, vol. 104 no. 5, pp 481-490, 2007.

[8] Hamilton III, H.R. and Brown, J., "CFRP Repair of Impact-Damaged Bridge Girders Volume II - Infrared Thermographic Inspection of FRP Composite Repairs," FDOT Report BC354 RPWO \#55, Florida Department of Transportation, 2004.

[9] Kharbari, V., Kaiser, H., Navada, R., Gosh, K., and Lee, L., "Methods for Detecting Defects in Composite Rehabilitated Concrete Structures," Final Report, SPR 336, Oregon Department of Transportation, 2005.

[10] Brown, J. and Hamilton III, H.R., "Quantitative Infrared Thermography Inspection for FRP Applied to Concrete Using Single Pixel Analysis," Construction \& Building Materials, ACI Committee 228 25th Anniversary Special Edition, Vol. 38, January 2013, pp. 1292-1301.

[11] Starnes, M. A., N. J. Carino, and Kausel, E.A., "Preliminary thermography studies for quality control of concrete structures strengthened with fiber-reinforced polymer composites." Journal of Materials in Civil Engineering 15(3): 266-273, (2003).

[12] J. Beck, B. Blackwell, and C. R. St. Clair, Inverse Heat Conduction: Ill-Posed Problems, Wiley Interscience, New York, 1985.

[13] J. C. Krapez and P. Cielo, Thermographic Nondestructive Evaluation: Data Inversion Procedures, Part I: 1-D Analysis, Res. Nondestr. Eval., vol. 3, pp. 81-100, 1991.

[14] A. Mandelis, Nondestructive Evaluation, PrenticeHall, Englewood Cliffs, NJ, 1994.

[15] C. K. Hsieh and K. C. Su, A Methodology of Predicting Cavity Geometry Based on the Scanned Surface Temperature Data-Prescribed Surface Temperature at the Cavity Side, J. Heat Transfer, vol. 102, pp. 324-329, 1980.

[16] A. G. Ramm, A Geometrical Inverse Problem, Inverse Problems, vol. 2, pp. L19-L21, 1986.

[17] A. J. Kassab, C. K. Hsieh, and J. Pollard, Solution of the Inverse Geometric Problem for the Detection of Subsurface Cavities by the IR CAT Method, in L. C. Wrobel and D. B. Ingham (eds.), Boundary Integral Formulations in Inverse Analysis, chap. 2, Computational Mechanics, Boston, 1997.

[18] G. S. Dulikravich and J. M. Martin, Geometrical Inverse Analysis Problems in Three- Dimensional Nonlinear Steady Heat Conduction, Eng. Anal. Boundary Elements, vol. 15, pp. 161-169, 1995.

[19] R. Bialecki, E. Divo, A. J. Kassab, and R. Ait Maalem Lahcen, Explicit Calculation of Smoothed Sensitivity Coefficients for Linear Problems, Int. J. Numer. Meth. Eng., vol. 57, no. 2, pp. 143-167, 2003.

[20] B. Blackwell, K. Dowding, and R. J. Chochran, Development and Implementation of Sensitivity Coefficient Equations for Heat Conduction Problems, Numer. Heat Transfer B, vol. 36, pp.15-32, 1999.

[21] C. A. Brebbia, J. C. F. Telles, and L. C. Wrobel, Boundary Element Techniques, Springer- Verlag, Berlin, 1984. 
[22] E. Divo and A. J. Kassab, Boundary Element Method for Heat Conduction with Applications in NonHomogeneous Media, Wessex Institute of Technology Press, Southampton and Boston, 2003.

[23] Kassab, A.J., Wrobel, L.C., Bialecki, R.A., and Divo, E., "Boundary Elements in Heat Transfer," Chapter 4 in Handbook of Numerical Heat Transfer, Minkowycz, W. and Sparrow, E.M. (eds.), John Wiley and Sons, New York, NY, John Wiley and Sons, Vol. 1, $2^{\text {nd }}$ Edition, pp. 125-166, 2005.

[24] E. Divo, A. J. Kassab and F. Rodriguez, An Efficient Singular Superposition Technique for Cavity Detection and Shape Optimization, Numerical Heat Transfer B, vol. 46, pp. 1-30, 2004.

[25] D. Ojeda, E. Divo and A. J. Kassab, Cavity Detection in Biomechanics by an Inverse Evolutionary Point Load BEM Technique, Inverse Problems in Science and Engineering, vol. 16, no. 8, pp. 981-993, 2008.

[26] Belytscho, T., Lu, Y.Y., and Gu, L., "Element-free Galerkin methods, Int. J. Num. Methods," Vol. 37, 1994, pp. 229-256.

[27] Atluri, S.N. and Shen, S., The Meshless Method, Tech. Science Press, Forsyth, 2002.

[28] Liu, G.R., Mesh Free Methods: Moving Beyond the Finite Element Method., CRC Press, Boca Raton, 2002.

[29] Gottlieb, D. and Orzag, S.A., Numerical Analysis of Spectral Methods: theory and applications, Society for Industrial and Applied Mathematics, Bristol, England, 1977.

[30] Maday, Y. and Quateroni, A., "Spectral and PseudoSpectral Approximations of the Navier-Stokes Equations," SIAM J. Numerical Analysis, 1982, Vol. 19, No. 4, pp. 761-780.

[31] Fasshauer, G, "RBF Collocation Methods as PseudoSpectral Methods," Boundary Elements XVII, Kassab, A., Brebbia, C.A. and Divo, E. (eds.), WIT Press, 2005, pp. 47-57.

[32] Powell, M.J.D., "The Theory of Radial Basis Function Approximation," in Advances in Numerical Analysis, Vol. II, Light, W., ed., Oxford Science Publications, Oxford, 1992. pp. 143-167.

[33] Buhmann, M.D., Cambridge Radial Basis Functions: Theory and Implementation University Press, Cambridge, 2003.

[34] Hardy, R.L., Multiquadric Equations of Topography and Other Irregular Surfaces, Journal of Geophysical Research, Vol. 176, pp. 1905-1915.

[35] Kansa, E.J., "Multiquadrics- a scattered data approximation scheme with applications to computational fluid dynamics I-surface approximations and partial derivative estimates," Comp. Math. Appl., Vol. 19, 1990, pp. 127-145.

[36] Franke, R., "Scattered data interpolation: Test of some methods," Math. Comput., Vol. 38, 1982, pp. 181-200.

[37] Cheng, A.H.-D., Golberg, M.A., Kansa, E.J., Zammito, G., "Exponential Convergence and $\mathrm{H}-\mathrm{c}$ Multiquadric Collocation Method for Partial Differential Equations," Numerical Methods in P.D.E., Vol. 19, No. 5, 2003, pp. 571-594.

[38] Sarler, B. and Vertnik, R., "Local Explicit Radial Basis Function Collocation Method for Diffusion
Problems," Computers and Mathematics with Applications,Vol. 51, No. 8, 2006, pp. 1269-1282.

[39] Divo, E. and Kassab, A.J., "A Meshless Method for Conjugate Heat Transfer Problems," Engineering Analysis, Vol. 29, No. 2, 2005, pp. 136-149.

[40] Divo, E. and Kassab, A.J., "An Efficient Localized RBF Meshless Method for Fluid Flow and Conjugate Heat Transfer," ASME Journal of Heat Transfer, 2007, Vol. 129, pp. 124-136.

[41] Divo, E. and Kassab, A.J., "Localized Meshless Modeling of Natural Convective Viscous Flows," Numerical Heat Transfer, Part A: Fundamentals, Volume 53, Issue 6, June 2008, pages 487-509.

[42] Kelly, J., Divo, E., and Kassab, A.J., "Numerical Solution of the Two-Phase Incompressible NavierStokes Equations Using a GPU-Accelerated Meshless Method," Engineering Analysis with Boundary Elements, 2012 (in review).

[43] Gerace, S., Erhart, K., Kassab, A.J., and Divo, E., "A Model-Integrated Localized Collocation Meshless Method for Large Scale Three-Dimensional Heat Transfer Problems," Engineering Analysis with Boundary Elements, 2012, (in press).

[44] Erhart, K. Kassab, A.J. and Divo, E., "An Inverse Localized Meshless Technique for the Determination of Non-Linear Heat Generation Rates in Living Tissues," International Journal of Numerical Methods for Heat and Fluid Flow, Vol. 18, No. 3/4, 2008, pp. 401-414.

[45] El Zahab, Z., Divo, E., and Kassab, A.J., "A Meshless CFD Approach for Evolutionary Shape Optimization of Bypass Grafts Anastomoses," Journal Inverse Problems in Science and Engineering, Vol. 17, No. 3, 411-435, 2009.

[46] A. Karageorghis, D. Lesnic and L Marin, A Survey of the MFS to Inverse Problems, Inverse Problems in Science and Engineering, vol. 19, no. 3, pp. 309-336, 2011.

[47] Hirt, C.W. and Nichols, B.D., Volume of Fluid (VOF) Method for the Dynamics of Free Boundaries, Journal of Computational Fluids, 1981, Vol. 39, pp. 201-225.

[48] Nelder, J.A. and Mead, R. A Simplex method for function minimization. Computer Journal, 7: 308-313, 1965. 